\title{
Stochastic Design Optimization of Microstructures with Utilization of a Linear Solver
}

\author{
Pinar Acar,* Siddhartha Srivastava, $\_$and Veera Sundararaghavan $\stackrel{ \pm}{ \pm}$ \\ University of Michigan, Ann Arbor, Michigan 48109
}

\begin{abstract}
DOI: $\underline{10.2514 / 1 . J 056000}$
Microstructure design can have a substantial effect on the performance of critical components in numerous aerospace applications. However, the stochastic nature of metallic microstructures leads to deviations in material properties from the design point, and it alters the performance of these critical components. In this work, an inverse stochastic design approach is introduced such that the material is optimized while accounting for the inherent variations in the microstructure. The highlight is an analytical uncertainty quantification model via a Gaussian distribution to model propagation of microstructural uncertainties to the properties. A metallic microstructure is represented using a finite element discretized form of the orientation distribution function. A stochastic optimization approach is proposed that employs the analytical model for uncertainty quantification, to maximize the yield strength of Galfenol microstructure in a compliant beam when constrained by uncertainties in the designed natural frequency of vibration. The results of the stochastic optimization approach are validated using a Monte Carlo simulation. It is also shown that multiple microstructure solutions can be identified using the null space of the linear systems involved in the optimization.
\end{abstract}

\section{Nomenclature}

$\begin{array}{ll}A & =\text { orientation distribution function } \\ C & =\text { coefficient matrix of multiple solution direct solver } \\ E_{1} & =\text { Young's modulus along axis } 1 \\ f & =\text { vector of objective function } \\ G_{12} & =\text { shear modulus in } 1-2 \text { plane } \\ I_{p} & =\text { polar inertia moment } \\ I_{1} & =\text { moment of inertia along axis } 1 \\ J & =\text { torsion constant } \\ k & =\text { number of orientation distribution functions } \\ L & =\text { beam length } \\ m & =\text { unit mass } \\ N & =\text { number of independent nodes } \\ n & =\text { axis of rotation } \\ n & =\text { number of null space vectors } \\ p_{1} & =\text { property vector for } S_{11} \\ p_{6} & =\text { property vector for } S_{66} \\ q & =\text { volume normalization vector } \\ r & =\text { orientation } \\ S & =\text { compliance } \\ t & =\text { computational time } \\ V & =\text { null space vector } \\ x & =\text { vector of unknowns } \\ \epsilon & =\text { volume-averaged strain } \\ \mu & =\text { mean value vector } \\ \Sigma & =\text { covariance matrix } \\ \sigma & =\text { volume-averaged stress } \\ \sigma_{y} & =\text { yield stress } \\ \sigma^{2} & =\text { variance } \\ \omega_{1 b} & =\text { first bending natural frequency } \\ \omega_{1 t} & =\text { first torsion natural frequency } \\ & \end{array}$

Presented as Paper 2017-1939 at the 58th AIAA/ASME/ASCE/AHS/ASC Structures, Structural Dynamics, and Materials Conference, Grapevine, TX, 9-13 January 2017; received 18 January 2017; accepted for publication 6 June 2017; published online 31 July 2017. Copyright () 2017 by Pinar Acar. Published by the American Institute of Aeronautics and Astronautics, Inc., with permission. All requests for copying and permission to reprint should be submitted to CCC at www.copyright.com; employ the ISSN 0001-1452 (print) or 1533-385X (online) to initiate your request. See also AIAA Rights and Permissions www.aiaa.org/randp.

*Graduate Research Assistant, Department of Aerospace Engineering. Student Member AIAA.

${ }^{\dagger}$ Graduate Research Assistant, Department of Aerospace Engineering.

\#Associate Professor, Department of Aerospace Engineering. Senior Member AIAA.

\section{Introduction}

$\mathbf{M}$ ICROSTRUCTURAL uncertainties arise from variations in manufacturing process conditions and can affect the performance of metallic materials in aerospace components. This is an aleatoric uncertainty, is unavoidable, and is naturally present in metallic systems. The present work aims to investigate the effect of aleatory uncertainties in microstructure modeling and the inverse design of stochastic microstructural features to achieve a prescribed statistical range of engineering properties. The current state of the art only addresses the direct uncertainty quantification problem (effect of uncertain microstructures on properties), and the stochastic inverse problem has not been addressed to the best of our knowledge. The direct problem has been generally addressed using computational techniques such as the Monte Carlo simulation (MCS), collocation, and spectral decomposition methods. Huyse and Maes [1] studied the effect of microstructural uncertainties on homogenized parameters by using random windows from the real microstructure, and they performed a MCS to identify the stochasticity in elastic parameters such as Young's modulus and Poisson's ratio. Sakata et al. [2] showed the variations in Young's modulus and Poisson's ratio due to microscopic uncertainties. They validated the results of their perturbation-based homogenization method with the MCS. In another paper, Sakata et al. [3] implemented a kriging approach to calculate the probability density functions of the material properties, and they used a MCS to study the uncertainties in geometry and the material properties of a microstructure through the same perturbation-based homogenization method. A computational stochastic modeling approach for random microstructure geometry was presented by Clement et al. [4,5]. The authors presented a highdimensional problem due to the high number of stochastic variables to represent the microstructure geometry. This high-dimensionality was reduced with implementation of polynomial chaos expansion. Creuziger et al. [6] examined the uncertainties in the orientation distribution function (ODF) values of a microstructure due to the variations in the pole figure values by using a MCS. Juan et al. [7] used a MCS to study effects of sampling strategy on the determination of various characteristic microstructure parameters, such as grain size distribution and grain topology distribution. Hiriyur et al. [8] studied an extended finite element method coupled with an $\mathrm{MCS}^{-}$approach to quantify the uncertainties in the homogenized effective elastic properties of multiphase materials. The uncertain parameters were assumed to be aspect ratios, spatial distribution, and orientation. They used a strain energy approach to analyze the uncertainties of the in-plane Young's modulus and Poisson's ratio. Kouchmeshky and Zabaras [9] presented propagation of initial texture and deformation process uncertainties on the final product properties. They used a data-driven approach to identify 
the joint probability distributions of random variables with a maximum entropy method, and they modeled the stochastic problem using a stochastic collocation approach. Madrid et al. [10] examined the variability and sensitivity of an in-plane Young's modulus of thin nickel polycrystalline films due to uncertainties in the microstructure geometry, crystallographic texture, and numerical values of single crystal elastic constants by using a numerical spectral technique. Niezgoda et al. [11] computed the variances of the microstructure properties by defining a stochastic process to represent the microstructure. They marked the sensitive regions in the convex hull that were generated with a principal component analysis, and they calculated the probability distributions of they stiffness and yield stress in cases of low, medium, and high variances.

These numerical uncertainty quantification techniques studied in the literature require high computational costs because they represent the joint probability distributions of the random variables using either interpolation functions or samples. As the problem complexity or the number of variables increases, the number of interpolation terms or sample points also increases. This is especially true for the ODFs that are discretized using finite element nodes or a spectral basis, and they contain a large number of free parameters for which the joint distribution needs to be sampled. Another drawback of the computational methods is the difficulty of satisfying design constraints such as volume fraction normalization. All these disadvantages imply the necessity of developing analytical solutions as a first step in uncertainty quantification. In this work, we present an analytical formulation based on a Gaussian distribution approach to represent the variations of the random parameters. The variations of the in-plane Young's modulus $E_{1}$ and the shear modulus $G_{12}$ are assumed to be provided by the manufacturer, and they are consistent with the Gaussian distribution. Then, the probability distributions of the ODFs are computed by solving an inverse problem. The variations in the compliance parameters, $S_{11}$ and $S_{66}$, are found first with transformation of the random variables rule by using input variations in $E_{1}$ and $G_{12}$. The compliance parameters are calculated first because they can be represented with linear equations in terms of the ODFs. The probability distributions of the compliance parameters are also assumed to be modeled with a Gaussian approach despite their nonlinear relation to $E_{1}$ and $G_{12}$ because the input uncertainties are very small. Then, the inverse problem to find the statistical properties of the ODFs is defined as a linear programming (LP) problem. A global stochastic optimization approach is implemented to this analytical solution framework to maximize the yield stress under vibration tuning constraints defined for the first bending and torsion natural frequencies of the cantilever beam. The optimization variables are defined as the inplane Young's modulus $E_{1}$ and they shear modulus $G_{12}$ of the galfenol material, and each design sample is assumed to have the same level of uncertainty. The LP problem approach has been studied before by Acar and Sundararaghavan to find the optimal processing route to produce a optimum microstructure design to the same vibration tuning problem [12]. However, the LP approach presented before was for the ODF solution of a deterministic system [12-14]. In this paper, we extend the LP solution methodology to identify the statistical parameters of the ODFs in the case of uncertainties in material properties. To the best of the authors' knowledge, this is the first analytical effort in the literature for quantification of microstructural stochasticity given the desired statistical range in properties: in effect, a stochastic inverse problem for microstructure design. The optimization results are also compared to the results of computational methods that employ a MCS to quantify the uncertainties. The analytical algorithm is able to compute the same optimization variables and a very close objective function value to the MCS solution while decreasing the computational time by almost two orders of magnitude. Once the optimum ODFs are achieved, then the multiple solution directions are identified using the direct linear solver, which was presented in our earlier works [12-14]. The linear solver is capable of finding exact solutions for multiple- and infinite-solution problems. The effect of uncertainties on the design objective is also discussed at the end by comparing the optimum results with the deterministic solution for the maximum yield stress. The organization of the paper is as follows: Section II addresses multiscale modeling of microstructures: particularly, the computation of volume-averaged properties. We introduce the analytical model for uncertainty quantification and a stochastic optimization approach in Sec. III. In Sec. IV, we report the results of the stochastic optimization studies performed using the analytical model and the MCS to quantify the uncertainties. A summary of the paper with potential future applications is presented in Sec. $\underline{\text { V. }}$

\section{Multiscale Modeling of Microstructures}

The alloy microstructure consists of multiple crystals, with each crystal having an orientation. The generalized Hooke's law for the aggregate of crystals may be written in the following form:

$$
<\boldsymbol{\epsilon}_{i j}>=\boldsymbol{S}_{i j k l}^{\mathrm{eff}}<\boldsymbol{\sigma}_{k l}>
$$

where $<\boldsymbol{\epsilon}_{i j}>$ and $<\boldsymbol{\sigma}_{k l}>$ are the volume-averaged strain and stress, respectively; and $S^{\text {eff }}$ is the effective compliance tensor in the coordinate system of the part. Assuming homogeneity of the deformation in a macroscale elementary volume, the effective elastic properties may be found through averaging using the Taylor approximation [15]:

$$
S^{\mathrm{eff}}=<S>
$$

If the effect of the factors (e.g., crystal size and shape) is ignored, averaging (denoted by $\langle\cdot\rangle$ in the preceding equation) can be performed over the ODF (represented by $\boldsymbol{A}$ ). The ODF gives the volume density of each orientation in the microstructure. If the orientation-dependent property for single crystals $\chi(r)$ is known, any polycrystal property can be expressed as an expected value, or average, given by the following:

$$
<\chi>=\int_{R} \chi(\boldsymbol{r}) \boldsymbol{A}(\boldsymbol{r}, \boldsymbol{t}) \mathrm{d} \boldsymbol{v}
$$

where the ODF $\boldsymbol{A}$ is a function of orientation $r$ and time $t$ (for plasticity problems). The average value is computed by integrating in the representative volume element $d v$, which can be obtained by considering the crystallographic symmetries.

The present work employs the axis-angle parameterization of the orientation space proposed by Rodrigues [16] because angle-axis representations define an alternate way of representing orientations compared to Euler angles $[\underline{17}, \underline{18}]$. The Rodrigues parameterization is created by scaling the axis of rotation $n$ as $r=n \tan (\theta / 2)$, where $\theta$ is the rotation angle. Finite element discretization of the orientation space and associated integration schemes using Gauss quadrature allow matrix representation of Eq. (3). The ODF is discretized into $N$ independent nodes with $N_{\text {elem }}$ finite elements and $N_{\text {int }}$ integration points per element. Using this parameterization, any polycrystal property can be expressed in a linear form as follows [19]:

$$
\begin{aligned}
<\chi> & =\int_{R} \chi(\boldsymbol{r}) A(\boldsymbol{r}, \boldsymbol{t}) \mathrm{d} \boldsymbol{v} \\
& =\sum_{n=1}^{N_{\text {elem }}} \sum_{m=1}^{N_{\text {int }}} \int_{R} \chi\left(\boldsymbol{r}_{m}\right) A\left(\boldsymbol{r}_{m}\right) w_{m}\left|J_{n}\right| \frac{1}{\left(1+\boldsymbol{r}_{m} \cdot \boldsymbol{r}_{m}\right)^{2}}
\end{aligned}
$$

where $A\left(r_{m}\right)$ is the value of the ODF at the $m$ th integration point with global coordinate $r_{m}$ of the $n$th element, $\left|J_{n}\right|$ is the Jacobian determinant of the $n$th element, $w_{m}$ is the integration weight associated with the $m$ th integration point, and

$$
\frac{1}{\left(1+r_{m} \cdot r_{m}\right)^{2}}
$$

represents the metric of the Rodrigues parameterization. This can be shown to be equivalent to an equation linear in the ODF: $<\chi>=\boldsymbol{p}^{T} \boldsymbol{A}$, where $\boldsymbol{A}$ is a vector containing the ODF values at the $k$ independent nodes of the ODF mesh [20]. In addition, the ODF is normalized to unity as $\boldsymbol{q}^{T} \boldsymbol{A}=1$ where $\boldsymbol{q}$ is a normalization vector. 


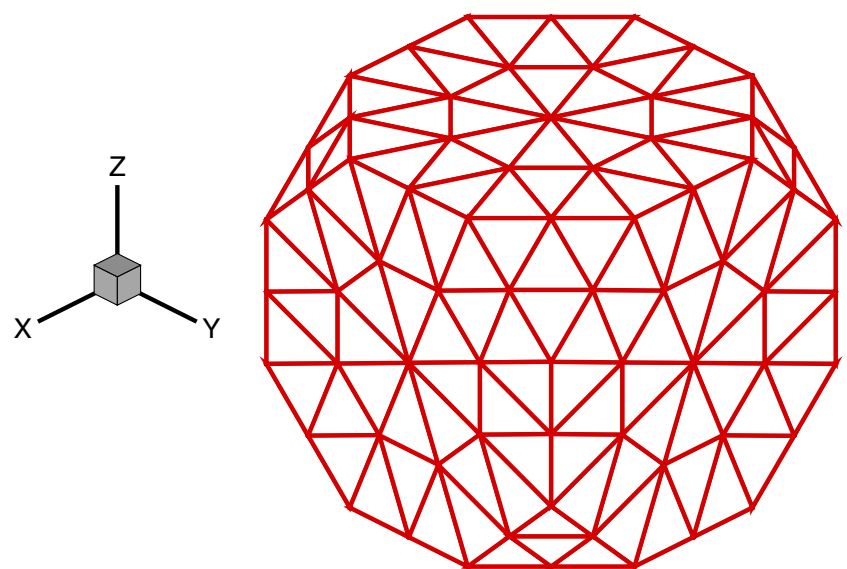

Fig. 1 ODF representation in the Rodrigues fundamental region for cubic crystal symmetry.

The polycrystal compliance $\bar{S}$ is computed through a weighted average (over $\boldsymbol{A}$ ) of the compliance values of individual crystals expressed in the sample reference frame using the lower bound approach (Reuss average). The yield stress is computed using a crystal plasticity model from recent work by Liu et al. [21]. The ODF representation for body-centered cubic (BCC) galfenol material is shown in Fig. 1.

\section{Stochastic Design Optimization of Microstructures}

\section{A. Problem Definition: Vibration Tuning for a Galfenol Beam}

The optimization problem studied in this paper aims to find the optimum microstructure design to maximize the yield stress of a cantilever beam made of galfenol under vibration tuning constraints (Fig. 2). The material properties of the galfenol beam are computed with the ODF values at independent nodal points. The same optimization problem was presented before [12-14] in the case of deterministic design variables. The vibration tuning constraints are defined for the first natural bending and torsion frequencies of the beam, which can be formalized in terms of the Young's modulus $E_{1}$ and the shear modulus $G_{12}$ for an orthotropic material:

$$
\begin{gathered}
\omega_{1 t}=\frac{\pi}{2 L} \sqrt{\frac{G_{12} J}{\rho I_{p}}} \\
\omega_{1 b}=(\alpha L)^{2} \sqrt{\frac{E_{1} I_{1}}{m L^{4}}} \text { and } \alpha L=1.87510
\end{gathered}
$$

where $G_{12}=1 / S_{66}, E_{1}=1 / S_{11}$, and $S_{11}$ and $S_{66}$ are the compliance elements. In these formulations, $J$ is a torsion constant, $\rho$ is the density, $I_{p}$ is the polar inertia moment, $m$ is the unit mass, $L$ is the length of the beam, and $I_{1}$ is the moment of inertia along axis 1 . To solve the problem, the length of the beam is taken as $L=0.45 \mathrm{~m}$ and the beam is considered to have a rectangular cross section with dimensions of $a=20 \mathrm{~mm}$ and $b=3 \mathrm{~mm}$.

\section{B. Analytical Model for Uncertainty Quantification}

The stochastic optimization approach presented in this work for vibration tuning of a galfenol beam microstructure starts with an assumption that we are given the variations of $E_{1}$ and $G_{12}$ parameters. According to this assumption, both $E_{1}$ and $G_{12}$ vary $\pm 5 \%$ around their mean values with respect to Gaussian distribution. The uncertainties of the ODFs are identified through an inverse design LP problem because compliances $S_{11}$ and $S_{66}$ can be defined with linear equations in terms of the ODFs using a lower bound average. However, the relation between the compliances and input parameters is nonlinear because $S_{11}=1 / E_{1}$ and $S_{66}=1 / G_{12}$. Similarly, the variables for the vibration tuning constraints, the first torsion natural frequency $\omega_{1 t}$, and the first bending natural frequency $\omega_{1 b}$ are nonlinear in terms of the input variables. The transformation of the random variables rule is used to identify the statistical properties for $S_{11}, S_{66}, \omega_{1 t}$, and $\omega_{1 b}$. Once the probability distributions of $S_{11}$ and $S_{66}$ are identified, the uncertainties in the ODF values are calculated using linear transformation. We assume that the probability distributions of the compliance parameters can be modeled with a Gaussian distribution approximation because the variations in the Gaussian input parameters $\left(E_{1}\right.$ and $\left.G_{12}\right)$ are small. The analytical modeling approaches used for computing linear and nonlinear material properties are summarized in the next sections.

\section{Uncertainties in Linear Material Properties}

The uncertainties in the linear material properties are computed using Gaussian distribution features in linear relations. The summary of the Gaussian approach to model a linear property is given in the following:

Assume a $d$-dimensional multivariate Gaussian distribution: $\boldsymbol{X} \sim \boldsymbol{N}_{d}(\boldsymbol{\mu}, \boldsymbol{\Sigma})$. Now, we define a new random variable:

$$
\boldsymbol{Z}=\boldsymbol{C X}
$$

where $C$ is a constant matrix. Then, $\boldsymbol{Z}$ is also Gaussian distributed [20]. The mean vector and covariance matrix of $\boldsymbol{Z}$ are given by the following:

$$
\begin{gathered}
\boldsymbol{\mu}_{Z}=C \boldsymbol{\mu}_{X} \\
\boldsymbol{\Sigma}_{Z}=C \boldsymbol{\Sigma}_{X} C^{T}
\end{gathered}
$$

The Gaussian approach presented here can be modified accordingly to represent the variations in the ODFs and linear material properties. Because the ODF values are identified from an inverse problem, we assume that the first $k-1$ number of ODFs are independent (where $k$ indicates the total number of ODF variables at independent nodes) to decrease the amount of random variables. To satisfy the unit volume fraction constraint, the equations to compute the statistical properties of the $k$ th ODF are modified. The mean and variance of the $k$ th ODF value can be obtained as

$$
\boldsymbol{E}\left[\boldsymbol{A}_{k}\right]=\boldsymbol{c}^{T} \boldsymbol{\mu}_{A}+\frac{1}{\boldsymbol{q}_{k}}
$$

and

$$
\boldsymbol{\sigma}^{2}\left[\boldsymbol{A}_{k}\right]=\boldsymbol{c}^{T} \boldsymbol{\Sigma}_{A} \boldsymbol{c}
$$

where $c_{i}=-\left(q_{i} / q_{k}\right), \mu_{A}=E\left(A_{i}\right)$, and

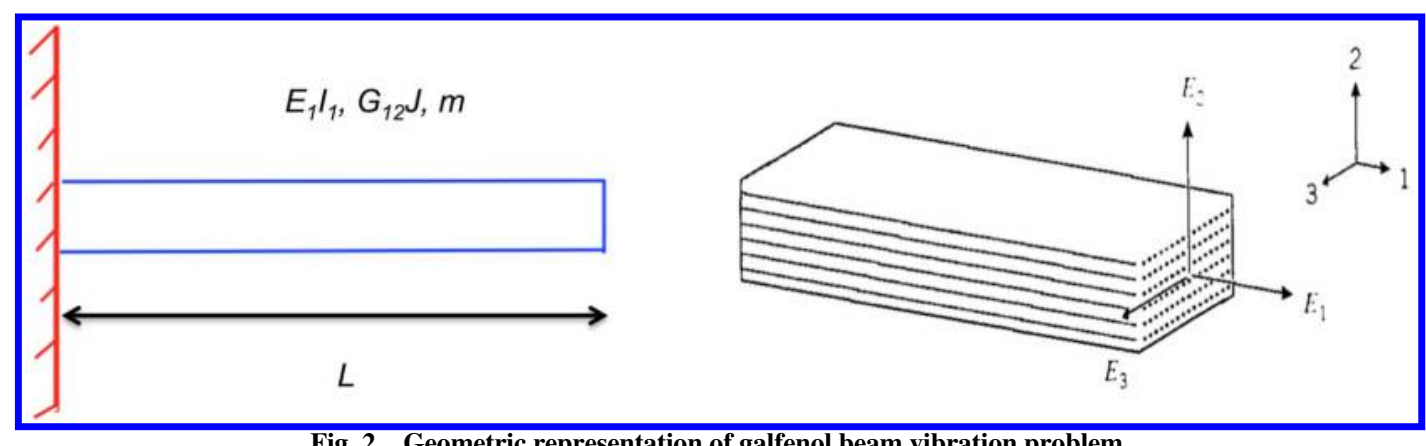

Fig. 2 Geometric representation of galfenol beam vibration problem. 


$$
\boldsymbol{\Sigma}_{A}=E\left[\left(A_{i}-\mu_{A_{i}}\right)\left(A_{j}-\mu_{A_{j}}\right)\right]
$$

After the modification for the $k$ th variable, the ODF covariance matrix can be written as follows:

$$
\boldsymbol{\Sigma}_{A}=\left[\begin{array}{cc}
\boldsymbol{\Sigma}_{A}^{*} & \boldsymbol{S} \\
\boldsymbol{S}^{T} & \sigma_{k}^{2}
\end{array}\right]
$$

where $\boldsymbol{\Sigma}_{A}^{*}$ is the covariance matrix defined for the first independent $k-1$ ODFs, and $S$ is a column vector for which the values are given by the following:

$$
\boldsymbol{S}_{i}=-\frac{1}{\boldsymbol{q}_{k}} \sum_{j=1}^{k-1} \boldsymbol{q}_{j}\left(\boldsymbol{\Sigma}_{A}^{*}\right)_{i j}
$$

The uncertainties in the linear material properties are computed using the linear transformation. The linear variables chosen for this study are the compliance parameters: $S_{11}$ and $S_{66}$. The mean and variance equations for $S_{11}$ can be shown as follows using the Gaussian approach. The same computation also applies to the statistical parameters of $S_{66}$ :

$$
\begin{gathered}
\boldsymbol{\mu}_{S_{11}}=\boldsymbol{p}_{1}^{T} \boldsymbol{\mu}_{A} \\
\boldsymbol{\sigma}_{S_{11}}^{2}=\boldsymbol{p}_{1} \boldsymbol{\Sigma}_{A} \boldsymbol{p}_{1}^{T}
\end{gathered}
$$

where $\boldsymbol{p}_{1}$ represents the single crystal property values for $\boldsymbol{S}_{11}$.

\section{Uncertainties in Nonlinear Material Properties}

The uncertainties in nonlinear material properties are computed using the transformation of random variables rule. The application of this rule is as follows: Given the input parameter $x$ and the output parameter $y$, we assume that the relation between $x$ and $y$ can be identified using $y=h(x)$ and can be inverted as $x=u(y)$. This method computes a Jacobian value $J$ based on this explicit relation (where $J=\mathrm{d} u / \mathrm{d} y$ ), and it finds the probability density function (PDF) of the output variable as a product of the input PDF and the Jacobian. Equation (14) shows the computation of the output PDF:

$$
f_{y}(y)=f_{x}[u(y)] \times|J|
$$

where $f_{x}$ and $f_{y}$ are the PDFs of input and output variables, respectively. Because the input $\operatorname{PDF} f_{x}$ and inverted function $u(y)$ are already known, the output PDF $f_{y}$ can be computed using this method. Then, the expected value $E[y]$ and variance $\sigma_{y}^{2}$ of the output parameter can be calculated using Eqs. (15) and (16), respectively [22]:

$$
\begin{gathered}
E[y]=\int_{y_{\min }}^{y_{\max }} y f_{y}(y) \mathrm{d} y \\
\sigma_{y}^{2}=E\left[(y-E[y])^{2}\right]
\end{gathered}
$$

where $y_{\min }$ and $y_{\max }$ are the minimum and maximum values the output variable $y$ can take. These values can be computed using the relation $y=h(x)$ for the minimum and maximum values of the input variable: $x_{\min }$ and $x_{\max }$, respectively. The approach is first applied to compute the PDF of the compliance parameters: $S_{11}=$ $1 / E_{1}$ and $S_{66}=1 / G_{12}$. The same method is then used to compute the PDFs of the first torsion and bending natural frequencies of a cantilever beam, $\omega_{1 t}$ and $\omega_{1 b}$, using Eqs. (ㅁ) and (ㅁ) .

\section{Linear Programming Approach for Inverse Design}

The statistical properties of the ODF values are identified by solving the inverse design problem as an LP problem. The PDFs of $S_{11}$ and $S_{66}$ were previously computed using the transformation of the random variables rule. The mean values and variances of $S_{11}$ and $S_{66}$ were then computed using Eqs. (15) and (16). The variations in these parameters were assumed to agree with the Gaussian distribution due to small variations in the input parameters, $E_{1}$ and $G_{12}$. With this assumption, the ODF values can be determined by solving an LP problem. A general formulation of an LP problem is given as follows:

$$
\begin{array}{r}
\min \boldsymbol{f}^{T} \boldsymbol{x} \\
\text { such that } \boldsymbol{A}_{\mathrm{eq}} \boldsymbol{x}=\boldsymbol{b} \text { eq } \\
\boldsymbol{A} \boldsymbol{x} \leq \boldsymbol{b} \\
\boldsymbol{l} \boldsymbol{b} \leq \boldsymbol{x} \leq \boldsymbol{u} \boldsymbol{b}
\end{array}
$$

The unknown vector $x$ of this LP problem includes the mean values and variances of the first $k-1$ ODF values: $\boldsymbol{\mu}_{A}$ and $\boldsymbol{\sigma}_{A}^{2}$. The mean and variance terms related to the $k$ th ODF value can then be obtained using the definitions for $\boldsymbol{\mu}_{A}$ and $\boldsymbol{\sigma}_{A}^{2}$ in the volume fraction normalization constraint equation. The equality constraints are derived by using the homogenized linear equations for the mean values [Eqs. (17) and (18)] and variances [Eqs. (19) and (20)]:

$$
\begin{gathered}
\boldsymbol{p}_{1}^{T} \boldsymbol{\mu}_{A}=\boldsymbol{\mu}_{S_{11}} \\
\boldsymbol{p}_{6}^{T} \boldsymbol{\mu}_{A}=\boldsymbol{\mu}_{S_{66}} \\
\boldsymbol{p}_{1} \boldsymbol{\Sigma}_{A} \boldsymbol{p}_{1}^{T}=\boldsymbol{\sigma}_{S_{11}}^{2} \\
\boldsymbol{p}_{6} \boldsymbol{\Sigma}_{A} \boldsymbol{p}_{6}^{T}=\boldsymbol{\sigma}_{S_{66}}^{2}
\end{gathered}
$$

In these equations, $\boldsymbol{p}_{1}$ and $\boldsymbol{p}_{6}$ are the vectors of length $k$ including single crystal coefficient values for $S_{11}$ and $S_{66}$, respectively; $\mu_{S_{11}}$ and $\mu_{S_{66}}$ are the mean values; and $\sigma_{S_{11}}^{2}$ and $\sigma_{S_{66}}^{2}$ are variances of $S_{11}$ and $S_{66}$. Accounting for the normalization constraint, we only solve for the $k-1$ ODF values. The augmented system of the equality constraints for the first $k-1$ ODF values can be derived as follows:

$\left[\begin{array}{cc}{\left[\boldsymbol{p}_{1}^{T}-\frac{\boldsymbol{p}_{1}(\boldsymbol{k})}{\boldsymbol{q}_{k}} \boldsymbol{q}^{T}\right]_{1 \times(k-1)}} & 0_{1 \times(k-1)} \\ {\left[\boldsymbol{p}_{6}^{T}-\frac{\boldsymbol{p}_{6}(\boldsymbol{k})}{\boldsymbol{q}_{k}} \boldsymbol{q}^{T}\right]_{1 \times(k-1)}} & 0_{1 \times(k-1)} \\ 0_{1 \times(k-1)} & \boldsymbol{P}_{1_{1 \times(k-1)}^{*}}^{*} \\ 0_{1 \times(k-1)} & \boldsymbol{P}_{6_{1 \times(k-1)}^{*}}^{*}\end{array}\right]\left[\begin{array}{l}\boldsymbol{\mu}_{A(k-1) \times 1} \\ \boldsymbol{\sigma}_{A(k-1) \times 1}^{2}\end{array}\right]=\left[\begin{array}{c}\boldsymbol{\mu}_{S_{11}}-\frac{\boldsymbol{p}_{1}(\boldsymbol{k})}{\boldsymbol{q}_{k}} \\ \boldsymbol{\mu}_{S_{66}}-\frac{\boldsymbol{p}_{6}(\boldsymbol{k})}{\boldsymbol{q}_{k}} \\ \boldsymbol{\sigma}_{S_{11}}^{2} \\ \boldsymbol{\sigma}_{S_{66}}^{2}\end{array}\right]$

where $q$ is a vector containing the first $k-1$ values of the normalization vector, and $0_{1 \times(k-1)}$ is a row vector of zeros with a length of $k-1$. The elements of the row vectors $\boldsymbol{P}_{1}^{*}$ and $\boldsymbol{P}_{6}^{*}$ can be calculated as follows by using Eqs. (19) and (20) with the definition for $\boldsymbol{\Sigma}_{A} \quad(i=1,2, \ldots, k-1):$

$$
P_{1}^{*}(i)=\left[p_{1}^{2}(i)+\left(p_{1}(k)+1\right)\left(p_{1}(i) c(i)\right)+\left(p_{1}^{2}(k) c^{2}(i)\right)\right]
$$

$$
P_{6}^{*}(i)=\left[p_{6}^{2}(i)+\left(p_{6}(k)+1\right)\left(p_{6}(i) c(i)\right)+\left(p_{6}^{2}(k) c^{2}(i)\right)\right]
$$

The first inequality equation is derived for the lower boundary of the $k$ th ODF value such that the first $k-1$ ODFs should satisfy the constraint $\boldsymbol{q}^{T} \boldsymbol{\mu}_{A} \leq 1$ to guarantee that the unit volume normalization constraint is satisfied with a nonnegative $k$ th ODF value $(\boldsymbol{q}>0$ and $\left.q_{k}>0\right)$. Because the compliance parameters are assumed to agree 
with the Gaussian approach, the ODF values have the same distribution because of their linear relation. We used the following inequalities to ensure that the probability distributions of the ODFs always satisfy the following nonnegativity condition: $-\boldsymbol{\mu}_{A}+\boldsymbol{z} \boldsymbol{\sigma}_{A}^{2} \leq 0$ and $-\boldsymbol{\mu}_{A_{k}}+\boldsymbol{z} \boldsymbol{\sigma}_{A_{k}}^{2} \leq 0$, where $z$ is a constant to be determined. In these inequality equations, the standard deviation parameter is approximated by the variance because the variances are the unknowns in the LP problem definition. The standard deviation can be replaced with the variance because the standard deviation and variance values of the compliances are in the same order, and the ODFs are assumed to follow the same trend. However, the variances are controlled with the constant parameter $z$ rather than directly considering the traditional $3.5 \sigma$ assumption for Gaussian distribution. The inequality equation for the variation of the $k$ th ODF can be manipulated further by using the definitions for $\mu_{A_{k}}$ and $\sigma_{A_{k}}^{2}$. The final forms of the inequality equations are given in Eqs. $(\underline{23}-\underline{25})$ :

$$
\begin{gathered}
\boldsymbol{q}^{T} \boldsymbol{\mu}_{A} \leq 1 \\
-\boldsymbol{\mu}_{A}+\boldsymbol{z} \boldsymbol{\sigma}_{A}^{2} \leq 0 \\
-\frac{1}{\boldsymbol{q}_{k}} \boldsymbol{q}^{T} \boldsymbol{\mu}_{A}+z C^{*} \boldsymbol{\sigma}_{A}^{2} \leq \frac{1}{\boldsymbol{q}_{k}}
\end{gathered}
$$

where the elements of the $C^{*}$ vector are $C^{*}(i)=c(i)^{2}$. Using Eqs. (23-25) the augmented system for the inequality constraints can be derived as follows:

$$
\left[\begin{array}{cc}
\boldsymbol{q}_{1 \times(k-1)}^{T} & 0_{1 \times(k-1)} \\
-[I]_{(k-1) \times(k-1)} & z[I]_{(k-1) \times(k-1)} \\
\frac{1}{\boldsymbol{q}_{k}} \boldsymbol{q}_{1 \times(k-1)}^{T} & \boldsymbol{z} C_{1 \times(k-1)}^{*}
\end{array}\right]\left[\begin{array}{c}
\boldsymbol{\mu}_{A(k-1) \times 1} \\
\boldsymbol{\sigma}_{A(k-1) \times 1}^{2}
\end{array}\right] \leq\left[\begin{array}{c}
1 \\
0_{(k-1) \times 1} \\
\frac{1}{\boldsymbol{q}_{k}}
\end{array}\right]
$$

where $[I]$ is the identity matrix. The objective of the stochastic optimization problem is to maximize the mean yield stress value of the beam. Because the standard LP problem defines the objective function for minimization instead of maximization, the negative of the yield stress value $-\sigma_{y}$ is minimized. This objective function is also linear in the ODFs such that

$$
-\boldsymbol{\sigma}_{y}=\left(-\boldsymbol{y}^{T}+\left(\frac{\boldsymbol{y}_{k}}{\boldsymbol{q}_{k}}\right) \boldsymbol{q}^{T}\right) \boldsymbol{\mu}_{A}-\frac{\boldsymbol{y}_{k}}{\boldsymbol{q}_{k}}
$$

where $y$ is the vector of yield stress coefficients for the first $k-1$ single crystals, and $y_{k}$ is the same coefficient value for the $k$ th single crystal. The objective function $f$ is defined as follows:

$$
\boldsymbol{f}=\left(-\boldsymbol{y}^{T}+\left(\frac{\boldsymbol{y}_{k}}{\boldsymbol{q}_{k}}\right) \boldsymbol{q}^{T}\right) \boldsymbol{\mu}_{A}
$$

and therefore $-\sigma_{y}=f-\left(y_{k} / q_{k}\right)$. The objective function of the LP problem for $\min \boldsymbol{f}^{T} \boldsymbol{x}$, can be written as follows:

$$
\boldsymbol{f}=\left[\boldsymbol{y}_{1 \times(k-1)}^{* T} 0_{1 \times(k-1)}\right]^{T}
$$

where $\boldsymbol{y}^{* T}$ is defined as follows:

$$
\boldsymbol{y}^{* T}=-\boldsymbol{y}^{T}+\left(\frac{\boldsymbol{y}_{k}}{\boldsymbol{q}_{k}}\right) \boldsymbol{q}^{T}
$$

In the final step, the lower and upper bounds are determined by considering the nonnegativity conditions for the ODFs. The unknowns of the LP problem, the mean values, and ondiagonal variance terms of the ODF parameters have a zero value lower bound. An ODF $A_{i}$ can have the value of $1 / q_{i}$ as an upper bound. This is also true for the mean values $\mu_{A_{i}}$. However, the variances are known to be lower than the mean values in this problem. Therefore, defining the same upper bound values for the corresponding variance terms is mathematically possible. The lower and upper bound vectors for this problem are then defined as $\boldsymbol{l} \boldsymbol{b}=\left[0_{1 \times 2(k-1)}\right]$ and $\boldsymbol{u} \boldsymbol{b}=\left[\begin{array}{ll}1 / \boldsymbol{q}_{i} & 1 / \boldsymbol{q}_{i}\end{array}\right]$, where $i=1,2, \ldots, k-1$.

\section{Definition of the Stochastic Optimization Problem}

The stochastic optimization problem for the vibration tuning of a galfenol beam microstructure with a yield stress objective is defined with the implementation of the presented analytical solution methodology for uncertainty quantification. The optimization starts with the global sampling for the input variables $\mu_{E_{1}}$ and $\mu_{G_{12}}$, which are the mean values of $E_{1}$ and $G_{12}$. In the next step, the statistical properties of compliances $S_{11}$ and $S_{66}$ and natural frequencies $\omega_{1 t}$ and $\omega_{1 b}$ are calculated using the random variables transformation rule in Sec. III.B.2. The ODF solution satisfying the calculated statistical properties of the compliances and maximizing the mean yield stress value is identified by implementing the LP problem of Sec. III.C to the optimization algorithm. The mathematical formulation of the optimization problem is given as follows:

$$
\max \mu_{\sigma_{y}}
$$

subject to

$$
P\left(20.25 \mathrm{~Hz} \leq \omega_{1 t} \leq 24.25 \mathrm{~Hz}\right)=1
$$

subject to

$$
P\left(132.75 \mathrm{~Hz} \leq \omega_{1 b} \leq 139.75 \mathrm{~Hz}\right)=1
$$

$$
s=\left(\mu_{E_{1}}, \mu_{G_{12}}\right)
$$

where the optimization variables are $\mu_{E_{1}}$ and $\mu_{G_{12}}$ in the global problem, as well as the means and variances of the first $k-1$ ODFs in the LP problem definition. Equation (27) shows the objective function, which is determined as the maximization of the mean yield stress value. The output variables have probability distributions based on their statistical properties. The constraint parameters are expected to satisfy the strict vibration tuning constraints in every point of their probability distribution. Therefore, the probability of satisfying the design constraints is expected to be one, as shown in Eqs. (28) and (29). In the last row, $s$ shows the vector of global optimization variables. The corresponding ODF solution to the optimum values of the global variables provides the optimal microstructure design of the problem. The nonnegativity condition of the ODFs is considered as a lower bound in the LP problem. The volume normalization constraint is also considered through the definition of the $k$ th ODF and the inequality constraint in Eq. (23).

\section{E. Multiple Solutions with a Direct Linear Solver}

After the computation of one optimum design, the multiple optimum ODF solutions to the galfenol problem are identified with the implementation of a direct linear solver through the use of linear parameters. These linear parameters are the optimum values of the compliance parameters and yield stress of the orthotropic galfenol beam, which can be computed through the optimum ODFs using the following equations:

$$
\begin{aligned}
& <\boldsymbol{S}^{*}>=\int \boldsymbol{S} \boldsymbol{A}^{*} \mathrm{~d} \boldsymbol{V} \\
& <\boldsymbol{\sigma}_{y}^{*}>=\int \boldsymbol{\sigma}_{y} \boldsymbol{A}^{*} \mathrm{~d} \boldsymbol{V}
\end{aligned}
$$

In Eqs. (31) and (32), $S^{*}$ and $\sigma_{y}^{*}$ are the optimum values for the compliance parameters and yield stress, respectively; and $\boldsymbol{A}^{*}$ denotes the vector of optimum ODF values. The objective of this step is to identify all the ODFs that provide the same $S^{*}$ and $\sigma_{y}^{*}$ values. 
Table 1 Stochastic optimization results for vibration tuning of the galfenol beam

\begin{tabular}{lcc}
\hline \hline Deterministic case & Stochastic (analytical) & Stochastic (MCS) \\
\hline$\sigma_{y}=367.9385 \mathrm{MPa}$ & $\mu_{\sigma_{y}}=340.1034 \mathrm{MPa}$ & $\mu_{\sigma_{y}}=340.2584 \mathrm{MPa}$ \\
$\omega_{1 t}=22.7038 \mathrm{~Hz}$ & $\mu_{\omega_{1 t}}=22.8272 \mathrm{~Hz}$ & $\mu_{\omega_{1 t}}=22.7408 \mathrm{~Hz}$ \\
$\omega_{1 b}=134.3167 \mathrm{~Hz}$ & $\mu_{\omega_{1 b}}=136.4554 \mathrm{~Hz}$ & $\mu_{\omega_{1 b}}=136.2892 \mathrm{~Hz}$ \\
$E_{1}=262.5002 \mathrm{GPa}$ & $\mu_{E_{1}}=270.3112 \mathrm{GPa}$ & $\mu_{E_{1}}=270.3112 \mathrm{GPa}$ \\
$G_{12}=87.5001 \mathrm{GPa}$ & $\mu_{G_{12}}=87.8067 \mathrm{GPa}$ & $\mu_{G_{12}}=87.8067 \mathrm{GPa}$ \\
$t=5 \mathrm{~min}$ & $t=20 \mathrm{~min}$ & $t=44$ hours $35 \mathrm{~min}$ \\
\hline \hline
\end{tabular}

The direct linear solver determines the multiple ODFs for the optimum properties. The solver is capable of finding multiple/infinite solutions because it uses the null space of the coefficient matrix to find the directions of the solutions. The use of the null space approach requires any one solution to the problem. This one solution comes from the global optimization result for this problem. The remaining infinite solutions are defined as the sums of this one solution and solution directions represented by null space vectors. The coefficient matrix can be defined using the linear relations for macroproperties and the unit volume fraction constraint for the ODF. The size of the coefficient matrix is $(4 \times k)$ because the rows are representing three independent linear equations for $S_{11}, S_{66}$, and $\sigma_{y}$ calculations, as well as one design constraint for volume fraction normalization $\left(\int \boldsymbol{A} \mathrm{d} \boldsymbol{V}=1\right)$.

Assuming $C$ is the coefficient matrix including the entries for compliances $S$ and yield stress $\sigma_{y}$, the infinite solutions can be represented as shown next $(i=1,2,3,4, \ldots, n)$ :

$$
\begin{gathered}
\boldsymbol{A}_{i}=\boldsymbol{A}_{1}+\lambda \boldsymbol{V}_{i} \\
\boldsymbol{V}_{i}=\operatorname{Null}(C(:, i))
\end{gathered}
$$

where Eq. (33) defines the infinite solutions $\boldsymbol{A}_{i}$ using one solution $\boldsymbol{A}_{1}$ and null space vectors $V_{i}$. Note that $n$ is the number of null space vectors. Even though the number of null space vectors is finite, the number of solutions can be infinite because $\lambda$ can be any number that satisfies the ODF positiveness constraint $(\boldsymbol{A} \geq 0)$. Because the optimization problem is solved in the space of macroproperties (property closure of homogenized parameters) and the space of macroproperties is generated by the ODF values through averaging equations, any point inside this solution domain corresponds to a known set of ODF values. Therefore, there is always at least one optimal ODF solution inside this domain. The solution strategy aims to find this optimum solution not only when it is unique but also when it is multiple. A more detailed discussion about implementation of the linear solver and generation of the property hulls can be found in earlier papers by Acar and Sundararaghavan [12-14].

\section{Results}

The stochastic optimization is performed using the incremental space filler (ISF) as the global sampling method for the input parameters and the nondominated sorting genetic algorithm (known as NSGA-II) as the optimization algorithm in modeFRONTIER software. To compare the effect of uncertainty to the final design and material properties, we also performed a deterministic optimization for the same problem. The constant parameter $z$ of the analytical LP approach is considered as $z=3.5$. In addition, we performed another stochastic optimization using the MCS method to model the uncertainties. In this MCS technique, we used 10,000 samples to generate the probability distributions for one set of global ISF sample points. The compliance values, $S_{11}$ and $S_{66}$, were calculated using the exact equations in terms of the input parameters. Then, the ODF solutions were identified by solving for 10,000 separate LP problems per one global sample. These deterministic LP problems were simplified forms of the presented LP methodology because they did not consider the inequality constraints defined for the variations [Eqs. (24) and (25)]. The MCS method, despite the use of the LP approach to solve the ODFs, was a computational burden compared to the required computational time to run the analytical solution. The optimum design parameters of stochastic optimization studies are given in Table 1. In all cases, the optimum parameters correspond to multiple optimal polycrystal designs with the implementation of the
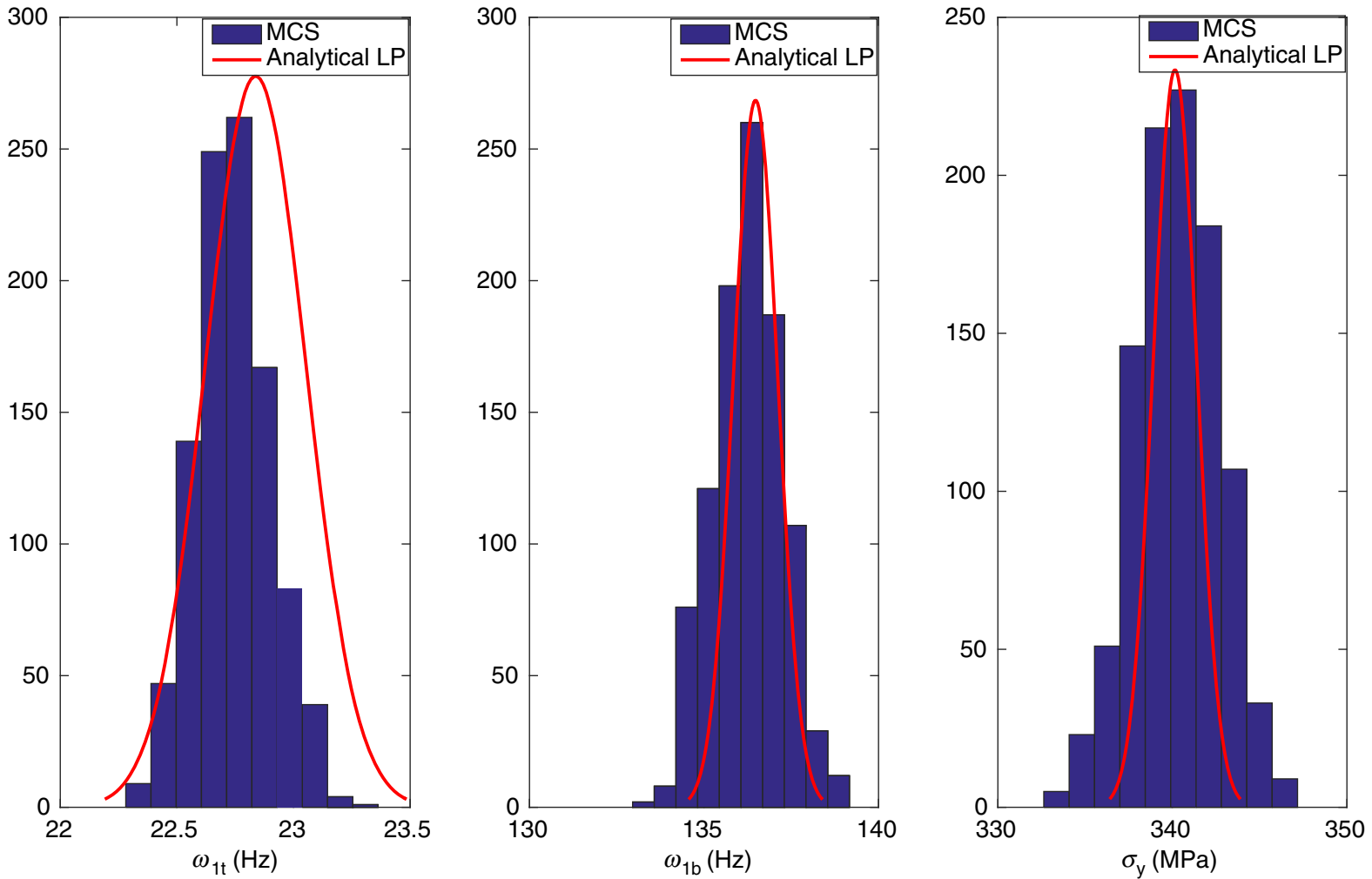

Fig. 3 Variations of yield stress and vibration frequencies of the stochastic optimum designs. 


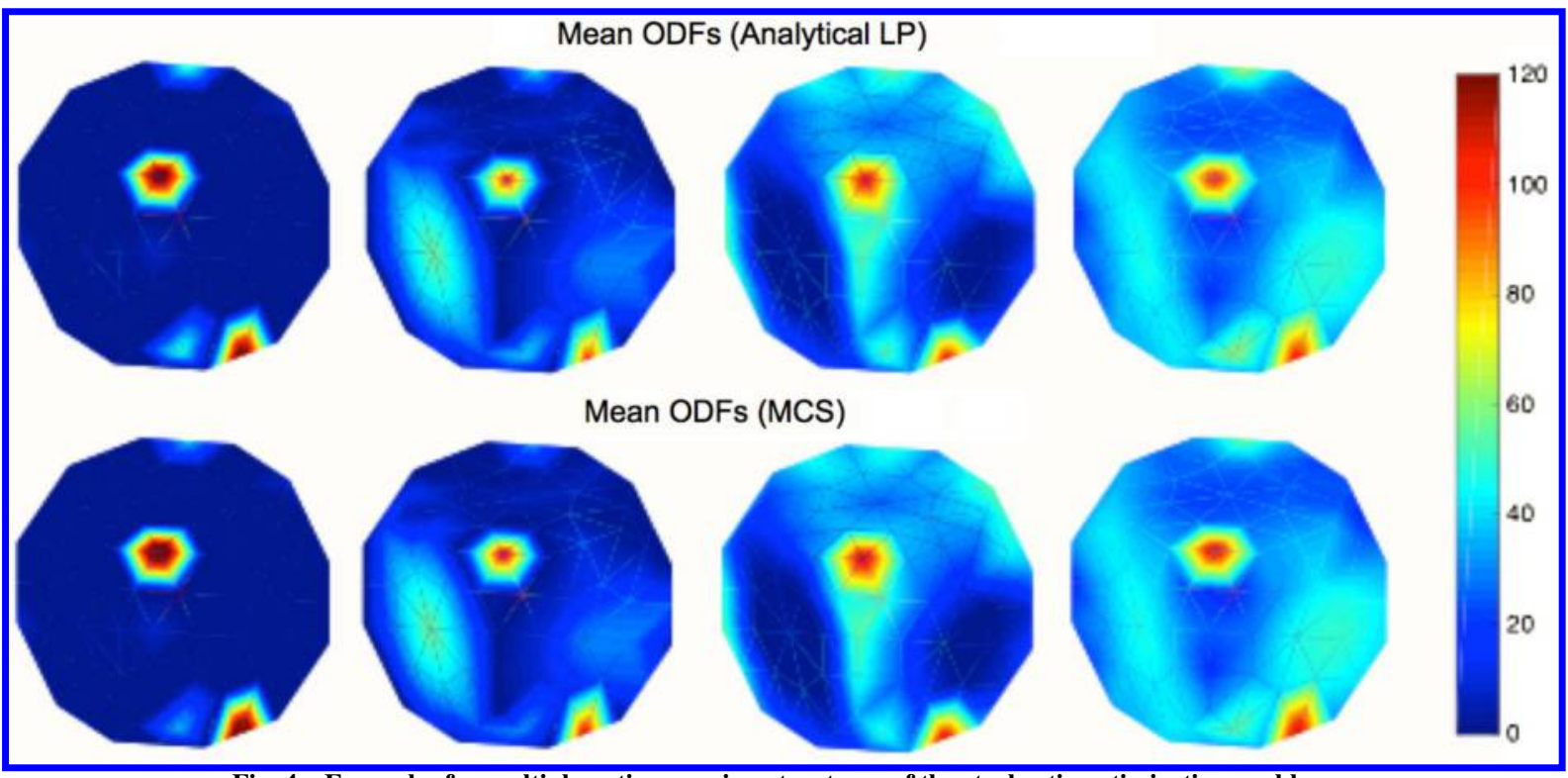

Fig. 4 Examples for multiple optimum microstructures of the stochastic optimization problem.

direct linear solver. The optimum deterministic parameters are also shown as the best case (with no uncertainties) in Table 1 to indicate the significant impact of the uncertainties to the design objective. The significant difference between the computational times spent on the stochastic optimization studies is also shown in the last row of Table 1 .

The difference between the optimum objective function values of the deterministic case and the stochastic optimization (Table 1) implies the substantial impact of the input uncertainties to the engineering properties. One critical feature of the results is that both stochastic optimization applications were able to identify the same solution for the global input parameters, $\mu_{E_{1}}$ and $\mu_{G_{12}}$. However, the optimum design criteria and objective function values are slightly different due to the different solution approaches in the analytical model, such as the random variables transformation rule and extended LP problem implementation by consideration of the ODF variances in contrast to the exact solution formulas being used by the MCS method. The variations of the yield stress and vibration frequencies of the stochastic optimum designs are shown in Fig. 3. According to the results in Fig. 3 , the analytical model is able to capture the values and variances of the optimum material properties.

After identifying the optimum solutions to the stochastic problems, the multiple polycrystal designs are also computed using the direct linear solution methodology with a null space approach. Some of the multiple optimum solutions to the ODF mean values obtained by the analytical model and MCS are shown in Fig. 4. The first microstructure design of both solutions is the optimum initial design identified with the global optimization. The other microstructures are obtained using the same independent null space vectors in the direct linear solver for both analytical and MCS solutions.

The small differences between the analytical model and MCS results in the final material properties shown in Fig. 3 and multiple optimum ODF solutions shown in Fig. 4 can be explained with two features of the analytical approach. First, the analytical solution assumes that the first $k-1$ ODFs are independent, and it identifies only the ondiagonal variances for these ODFs. The system of equations in the LP problem already implies an underdetermined system, and the consideration of the nondiagonal terms makes the solution infeasible. However, the MCS method automatically considers the dependencies of the ODFs because it uses the exact solutions with direct sampling. The other reason is predicted to be the effect of the adjustable constant parameter $z$ of the analytical solution, which represents the ODF variations. We used $z=3.5$ in the results reported in Figs. $\underline{3}$ and $\underline{4}$. The effect of this parameter is further investigated by computing the yield stress values of the optimum microstructure using different $z$ values. The same analysis is not

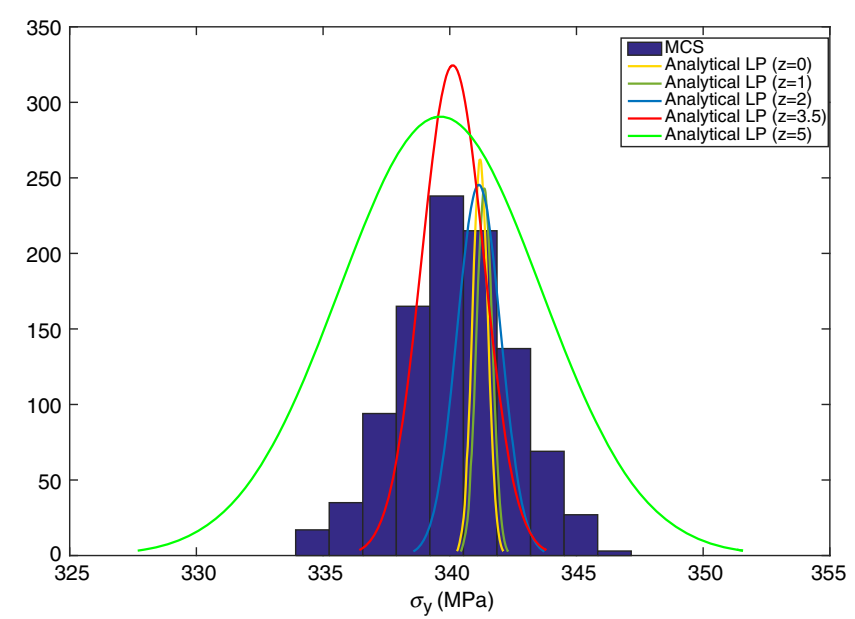

Fig. 5 Yield stress distributions of the optimum microstructure design with varying $z$ values in the analytical solution.

performed for the natural frequency parameters because they are directly related to the global variables, and not to the LP problem, so the change in $z$ parameter does not affect them. The yield stress distributions of the optimum microstructure design with varying $z$ values in the analytical solution are shown in Fig. 5.

Figure $\underline{5}$ implies that the variations in the optimum yield stress parameter are smaller when $z$ is smaller. This is an expected result because $z$ represents the variations in the ODFs. Compared to the MCS samples, the best matching analytical result is provided by the $z=3.5$ condition, which was also used in the stochastic optimization.

\section{Conclusions}

The present work addresses a stochastic optimization problem that employs an analytical uncertainty modeling approach. The optimization problem is solved to maximize the mean value of the yield stress of a galfenol beam under vibration tuning constraints defined for the first torsion and bending natural frequencies. It was initially assumed that the probability distributions of the Young's modulus and shear modulus parameters $\left(E_{1}\right.$ and $\left.G_{12}\right)$ were provided. The probability distributions of these input parameters were assumed to be Gaussian with $\pm 5 \%$ variations around the mean value. For vibration tuning constraints, the random variables transformation rule was applied to compute the probability distributions of the first torsion and bending natural frequencies of the beam. To compute 
the probability distributions of the orientation distribution function values, the statistical properties of the compliances, $S_{11}$ and $S_{66}$, were first computed using the same random variables transformation technique. It was assumed that the probability distributions of the compliance parameters could be modeled with a Gaussian approach because the input uncertainties were small. Next, an inverse problem was solved to identify the mean and variances of the orientation distribution function (ODF) parameters. The inverse design problem was solved by implementing a linear programming problem approach because the equations to compute the compliance parameters and yield stress were linear in terms of the ODFs. The values for the first $k-1$ ODF parameters were computed, and the $k$ th ODF was identified through the implementation of the volume fraction normalization constraint to the linear programming (LP) problem. The stochastic optimization was performed on this analytical model to find the optimal ODF solution that maximized the mean yield stress value. Another stochastic optimization was also performed that used a Monte Carlo simulation method to model the uncertainties. The analytical solution for uncertainty modeling not only reduced the computational time requirement for the optimization but also provided the same optimum parameters with very slight differences in yield stress and frequency parameters as compared to the Monte Carlo simulation results. A deterministic optimization was also performed to compare the optimum results with and without the effect of uncertainties. The differences on the optimum solutions of the deterministic and stochastic cases implied the necessity of considering uncertainties when modeling the materials. The multiple optimal microstructure designs were also identified by using a direct linear solver with a null space approach. Finally, a parametric study was performed to analyze the mathematical definition of the ODF variations in the LP problem and its effect on the optimum result. A future effort will aim to improve the analytical solution methodology to solve the ODF parameters without the independency assumption.

\section{References}

[1] Huyse, L., and Maes, M. A., "Random Field Modeling of Elastic Properties Using Homogenization," Journal of Engineering Mechanics, Vol. 127, No. 1, 2001, pp. 27-36. doi:10.1061/(ASCE)0733-9399(2001)127:1(27)

[2] Sakata, S., Ashida, F., Kojima, T., and Zako, M., "Three-Dimensional Stochastic Analysis Using a Perturbation-Based Homogenization Method for Elastic Properties of Composite Material Considering Microscopic Uncertainty," International Journal of Solids and Structures, Vol. 45, Nos. 3-4, 2008, pp. 894-907. doi:10.1016/j.ijsolstr.2007.09.008

-[3] Sakata, S., Ashida, F., and Zako, M., "Kriging-Based Approximate Stochastic Homogenization Analysis for Composite Materials," Computer Methods in Applied Mechanics and Engineering, Vol. 197, Nos. 21-24, 2008, pp. 1953-1964. doi:10.1016/j.cma.2007.12.011

[4] Clement, A., Soize, C., and Yvonnet, J., "Computational Nonlinear Stochastic Homogenization Using a Nonconcurrent Multiscale Approach for Hyperelastic Heterogeneous Microstructure Analysis," International Journal for Numerical Methods in Engineering, Vol. 91, No. 8, 2012, pp. 799-824. doi:10.1002/nme.v91.8

-[5] Clement, A., Soize, C., and Yvonnet, J., "Uncertainty Quantification in Computational Stochastic Multi-Scale Analysis of Nonlinear Elastic Materials," Computer Methods in Applied Mechanics and Engineering, Vol. 254, Feb. 2013, pp. 61-82. doi:10.1016/j.cma.2012.10.016
[6] Creuziger, A., Syed, K., and Gnaupel-Herold, T., "Measurement of Uncertainty in Orientation Distribution Function Calculations," Scripta Materialia, Vols. 72-73, Feb. 2014, pp. 55-58. doi:10.1016/j.scriptamat.2013.10.017

[7] Juan, L., Liu, G., Wang, H., and Ullah, A., "On the Sampling of Three-Dimensional Polycrystalline Microstructures for Distribution Determination," Journal of Microscopy, Vol. 44, No. 2, 2011, pp. 214-222.

[8] Hiriyur, B., Waisman, H., and Deodatis, G., "Uncertainty Quantification in Homogenization of Heterogeneous Microstructures Modeled by XFEM," International Journal for Numerical Methods in Engineering, Vol. 88, No. 3, 2011, pp. 257-278. doi: $10.1002 / \mathrm{nme} .3174$

[9] Kouchmeshky, B., and Zabaras, N., "The Effect of Multiple Sources of Uncertainty on the Convex Hull of Material Properties of Polycrystals," Computational Materials Science, Vol. 47, No. 2, 2009, pp. 342-352. doi:10.1016/j.commatsci.2009.08.010

[10] Madrid, P. J., Sulsky, D., and Lebensohn, R. A., "Uncertainty Quantification in Prediction of the In-Plane Young's Modulus of Thin Films with Fiber Texture," Journal of Microelectromechanical Systems, Vol. 23, No. 2, 2014, pp. 380-390. doi:10.1109/JMEMS.2013.2279500

[11] Niezgoda, S. R., Yabansu, Y., and Kalidindi, S. R., "Understanding and Visualizing Microstructure and Microstructure Variance as a Stochastic Process," Acta Materialia, Vol. 59, No. 16, 2011, pp. 6387-6400. doi:10.1016/j.actamat.2011.06.051

[12] Acar, P., and Sundararaghavan, V., "Linear Solution Scheme for Microstructure Design with Process Constraints," AIAA Journal, Vol. 54, No. 12, 2016, pp. 4022-4031 .

[13] Acar, P., and Sundararaghavan, V., "Utilization of a Linear Solver for Multiscale Design and Optimization of Microstructures in an Airframe Panel Buckling Problem," 57th AIAA/ASCE/AHS/ASC Structures, Structural Dynamics, and Materials Conference, AIAA Paper 20160156, Jan. 2016.

[14] Acar, P., and Sundararaghavan, V., "Utilization of a Linear Solver for Multiscale Design and Optimization of Microstructures," AIAA Journal, Vol. 54, No. 5, 2016, pp. 1751-1759. doi:10.2514/1.J054822

[15] Taylor, G. I., "Plastic Strain in Metals," Journal of the Institute of Metals, Vol. 62, May 1938, pp. 307-324.

[16] Kumar, A., and Dawson, P. R., "Computational Modeling of F.C.C. Deformation Textures over Rodrigues' Space," Acta Materialia, Vol. 48, No. 10, 2000, pp. 2719-2736. doi:10.1016/S1359-6454(00)00044-6

[17] Bunge, H. J., Texture Analysis in Materials Science, Butterworths, London, 1982, pp. 3-41.

[18] Wenk, H. R., Preferred Orientation in Deformed Metals and Rocks, Academic Press, London, U.K., 1985, pp. 73-108.

-[19] Sundararaghavan, V., and Zabaras, N., "Linear Analysis of Texture-Property Relationships Using Process-Based Representations of Rodrigues Space," Acta Materialia, Vol. 55, No. 5, 2007, pp. $1573-1587$. doi:10.1016/j.actamat.2006.10.019

[20] Acar, P., and Sundararaghavan, V., "Uncertainty Quantification of Microstructural Properties due to Variability in Measured Pole Figures," Acta Materialia, Vol. 124, Feb. 2017, pp. 100-108 .

[21] Liu, R., Kumar, A., Chen, Z., Agrawal, A., Sundararaghavan, V., and Choudhary, A., "A Predictive Machine Learning Approach for Microstructure Optimization and Materials Design," Nature Scientific Reports, Vol. 5, June 2015, Paper 11551.

[22] Ross, S. M., Introduction to Probability Models, 10th ed., Elsevier, New York, 2010, pp. 40, 43. 
This article has been cited by:

1. Pinar Acar, Veeraraghavan Sundararaghavan. Replacing Microstructural Experiments with Reconstruction Algorithms: Do Epistemic Uncertainties Allow? . [Citation] [PDF] [PDF Plus] 